\title{
A method to measure nanomechanical properties of biological objects
}

\author{
Nicoleta Ploscariu and Robert Szoszkiewicz ${ }^{\text {a) }}$ \\ Department of Physics, Kansas State University, Manhattan, Kansas 66506, USA
}

(Received 5 September 2013; accepted 8 December 2013; published online 30 December 2013)

\begin{abstract}
We postulate that one will be able to quantitatively infer changes in the mechanical properties of proteins, cells, and other biological objects (BO) by measuring the shifts of several thermally excited resonance frequencies of atomic force microscopy cantilevers in contact with BOs. Here, we provide a method to extract spring constants and molecular damping factors of BOs in biologically relevant phosphate buffered saline medium and using compliant AFM cantilevers with a small aspect ratio (a ratio of length to width). (C) 2013 AIP Publishing LLC.

[http://dx.doi.org/10.1063/1.4858411]
\end{abstract}

Key processes related to development and tissue homeostasis depend on mechanical properties of the involved proteins, cells, and other biological objects (BO). ${ }^{1-6}$ It has become possible to interrogate such processes in situ and with a spatial resolution down to a single molecule. ${ }^{1,7}$ Quantitative, fast, and non-destructive nanomechanical measurements of BOs are becoming possible too. For example, one can learn about forces associated with major conformational transitions during mechanical stretching of single proteins using optical and magnetic tweezers and atomic force microscopy (AFM). ${ }^{8-11}$

Recent advances in high bandwidth AFM and compliant low-drift AFM cantilevers make it possible to visualize, manipulate, and indent single proteins, biological cells, and their films. $8,11,12$ Calibrated AFM force-distance curves yield contact stiffness or elastic modulus of BOs. ${ }^{13,14}$ Techniques utilizing small-amplitude vibrations of the AFM cantilevers provide elastic moduli of agglomerated proteins and single cells non-destructively. ${ }^{15,16}$ Use of ultrasonic techniques for nanomechanical measurements additionally eliminates mechanical hysteresis of the AFM cantilevers. ${ }^{17}$

Exploitation of a multi-frequency response of the AFM cantilever is expected to provide many topographical and nanomechanical parameters simultaneously and quickly. ${ }^{18}$ Bimodal AFM methods have been already implemented. ${ }^{18-20}$ These methods measure amplitudes and phases of the first two flexural resonance modes of the vibrating AFM cantilever in intermittent contact with the sample. The amplitudes and phases are manipulated to produce the maps of local stiffness, stiffness gradient, and the viscoelastic dissipation in contact with cells and protein films. Similar approaches have been also applied to torsional excitations of the AFM cantilevers. ${ }^{21}$ While multifrequency AFM is highly accurate in theory, complicated and highly non-linear dependencies of the amplitudes and phases with measured tip-sample distance as well as their couplings can produce experimental artifacts. ${ }^{18,20,22}$ Thus, complementary approaches to obtain quick and complete nanomechanical characterization of BOs are desirable.

We propose to measure stiffness and other nanomechanical properties of a $\mathrm{BO}$ from the shifts of the resonance

\footnotetext{
${ }^{\text {a) }}$ Author to whom correspondence should be addressed. Electronic mail: szosz@ksu.edu
}

frequencies for a thermally excited AFM cantilever in contact with such an object. The number of simultaneously elucidated nanomechanical parameters depends only on the number of the resonances measured, i.e., electronics AFM bandwidth. ${ }^{23}$

Using a similar approach, Dupas et al. ${ }^{24}$ elucidated local stiffness and internal friction of some engineering materials. However, while measurements on engineering samples use stiff AFM cantilevers in air, the measurements on biological entities need to use compliant AFM cantilevers in biological media. For cantilevers with small aspect ratio, problems are exacerbated due to issues in providing analytical description of the hydrodynamic flow. ${ }^{25}$ Currently, such cantilevers are among the most appropriate ones for probing compliant BOs. Thus, a comprehensive approach needs to be developed to accurately fit flexural resonances of compliant AFM cantilevers with a small aspect ratio ${ }^{26}$ in contact with biological specimens in dissipative media.

In this letter, we develop a method to fit multiple resonance frequencies for compliant AFM cantilevers with a small aspect ratio in the biologically relevant phosphate buffered saline (PBS) buffer. The cantilevers are clamped on one end with the other end free. For each cantilever, we obtain geometrical and material properties. Properties with largest uncertainties, e.g., thickness, are determined from the fit of several consecutive resonance frequencies in air. Other geometrical and material parameters are measured or calculated. To fit resonances in air, we use the model of Dupas et al $^{24}$ developed for a free cantilever in vacuum. We obtain satisfactory agreement between fitted and measured resonances in air. Better agreement is obtained, when we correct the model of Dupas et al. for air damping using the results of Sader. ${ }^{27}$ These developments are a starting point to fit the resonance frequencies of the cantilevers in the PBS buffer and introduce corrections to properly account for the hydrodynamic flow. We introduce a generalized hydrodynamic function, which we obtain from a set of several cantilevers. We apply our model to obtain shifts in resonance frequencies expected in contact between a cantilever and a protein sample, and provide an error progression analysis.

We use Olympus AFM biolevers model BL-RC150VB, type "B," in air and in Dulbecco's PBS buffer $(137 \mathrm{mM} \mathrm{NaCl}$, $3 \mathrm{mM} \mathrm{KCl}, 2 \mathrm{mM} \mathrm{K \textrm {K } _ { 2 }} \mathrm{PO}_{4}$, and $8 \mathrm{mM} \mathrm{Na} 2 \mathrm{HPO}_{4} \cdot 7 \mathrm{H}_{2} \mathrm{O}$ ) from Midsci, USA. Thermal deflection signal of freely 
vibrating AFM cantilevers is fast Fourier transformed to produce amplitude spectra using our custom AFM setup as described in Ref. 28. Resonance frequencies from the cantilever's amplitude spectra are read using multipeak fit package with Voigt model in Igor Pro, Wavemetrics, USA. We fit the resonance frequencies using procedures written in Igor. Fit errors are the relative errors between fitted and measured resonance frequencies. ${ }^{29}$ The electronics bandwidth is $250 \mathrm{kHz} .^{28}$

Fig. 1 shows an AFM cantilever as a rectangular EulerBernoulli beam interacting with an arbitrary body. The cantilever has length $L$, width $b$, thickness $t$, density $\rho$, Young's modulus $E$, tip length $h_{t i p}$, and tip mass $m_{t i p}$ attached at a point $\beta L$ along the beam. The cantilever is tilted at an angle $\alpha$ with respect to the normal to the substrate. One beam end is clamped by a support spring with an elastic spring constant $k_{S}$. The other end is either left free or in contact-via its tip-with a body of interest. The body of interest is abstracted by an ensemble of dissipative springs providing its mechanical signature. We use the Kelvin-Voigt model, where spring constants $k$ are in parallel with their corresponding molecular damping factors $\gamma$. BOs and proteins, in particular, exhibit distinctively different visco-elastic properties along each pulling/pushing direction. ${ }^{30,31}$ Thus, in Fig. 1, we adopt only a reduced mechanical signature with two dissipative and mutually perpendicular springs: one along a normal force-exerting direction with $k_{n}$ and $\gamma_{n}$, and the other with $k_{\text {lat }}$ and $\gamma_{\text {lat }}{ }^{32}$

Dupas et al..$^{24}$ showed how to obtain the values of $k$ and $\gamma$ analytically for the cantilever in contact with a viscoelastic body as in Fig. 1 and obeying an equation of a moving Euler-Bernoulli beam

$$
E I \frac{\partial^{4} y}{\partial x^{4}}+\mu \frac{\partial^{2} y}{\partial t^{2}}=0
$$

Here, $I$ is the areal moment of inertia, $y$ is the vertical deflection, and $\mu$ is the mass of the cantilever over its length. The solution of Eq. (1) is of the form

$$
y(x, t)=y(x) \exp (i \omega t),
$$

with $y(x)$ of the form

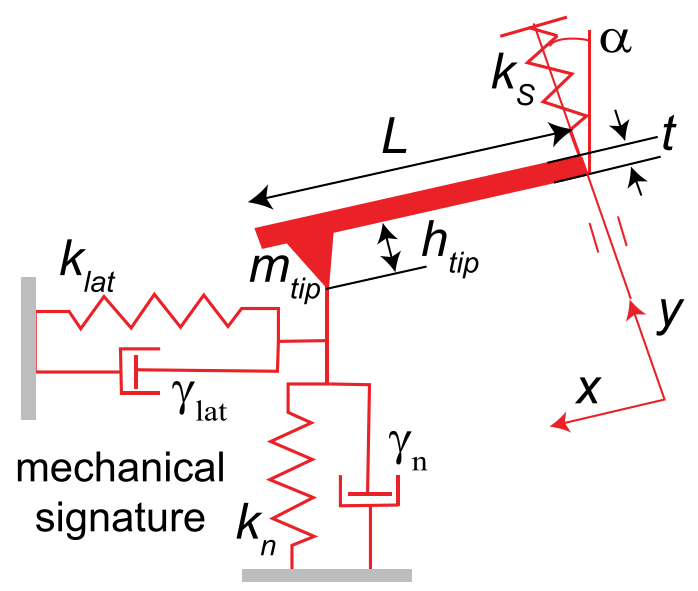

FIG. 1. Adapted model to obtain mechanical signatures of a biological object in contact with an AFM cantilever.

$$
\begin{aligned}
y(x)= & A_{1}[\cos (\kappa x)+\cosh (\kappa x)]+A_{2}[\cos (\kappa x)-\cosh (\kappa x)] \\
& +A_{3}[\sin (\kappa x)-\sinh (\kappa x)]+A_{4}[\sin (\kappa x)-\sinh (\kappa x)] .
\end{aligned}
$$

Here: $\omega$ is an angular frequency, $\kappa$ is a wave vector, and parameters $A_{1}-A_{4}$ are obtained from boundary conditions. Equations (1)-(3) extend to any $\mathrm{BO}$ provided that an accurate model for the cantilever is developed in appropriate media.

Fig. 2 presents a typical amplitude vs. frequency spectrum for the BL-RC150VB cantilever obtained from its thermal excitations in air. ${ }^{28}$ Three flexural resonances at frequencies of $11.6 \mathrm{kHz}, 76.1 \mathrm{kHz}$, and $219 \mathrm{kHz}$ are fitted using the model of Dupas et al., which depends on the following variables: $L, b, t, \beta, h_{t i p}, m_{t i p}, k_{S}, \alpha, \epsilon, E, a_{0}$. Here, $\epsilon$ is a mean position of the laser beam on the AFM cantilever, and $a_{0}$ is the cantilever's excitation amplitude. In order to get an accurate agreement between measured and modeled resonance frequencies, we fit only the values of $t, \beta, h_{t i p}$, and $E$, which have the largest uncertainties, and treat the other variables as parameters. We also constrain the four variables as follows. The values of $\beta$ are estimated from optical images of the AFM cantilevers and constrained to $0.95 \pm 0.05$. Similarly, the values of $h_{\text {tip }}$ are constrained to $7.5 \pm 2.5 \mu \mathrm{m}$. Using the manufacturer's scanning electron microscopy (SEM) measurements, the cantilever's thickness is constrained to $200 \pm 26 \mathrm{~nm}$, and the value of $E$ to $155 \pm 10 \mathrm{GPa}^{34-36}$

The cantilever's density $\rho$ is related to thickness using a weighted average with the density of silicon nitride $\rho_{\text {SiNx }}=3100 \mathrm{~kg} / \mathrm{m}^{3},{ }^{33}$ the density of the $10 \mathrm{~nm}$ chromium layer $\rho_{C r}=7140 \mathrm{~kg} / \mathrm{m}^{3}$, and the density of the $50 \mathrm{~nm}$ gold coating $\rho_{A u}=19320 \mathrm{~kg} / \mathrm{m}^{3}$. The values of $L$ and $b$ are obtained within $1 \%$ and $2 \%$ relative errors, respectively, using optical microscopy. ${ }^{35}$ The value of $b$ is further constrained through measurements of the torsional resonance frequencies, when they are visible on the amplitude spectra. ${ }^{37}$ Using the manufactures' SEM images of the tips, the value of $m_{\text {tip }}$ is calculated supposing that a tip is half of a pyramidal shell with thickness $t .^{38}$ The value of $k_{S}=600 \pm 30 \mathrm{~N} / \mathrm{m}$ is measured using a dynamometer. ${ }^{39}$ Since only resonance frequencies are fitted, and not their shape, the values of $\epsilon$ and $a_{0}$ are arbitrary selected as $0.8 L$, and $10^{-22} \mathrm{~m}$, respectively. ${ }^{24}$ The values of $k_{n}=k_{\text {lat }}=\gamma_{n}=\gamma_{\text {lat }}=0$.

The model of Dupas et al. produces a reasonable fit in Fig. 2 with an accumulated error of $3.4 \%$ over three flexural

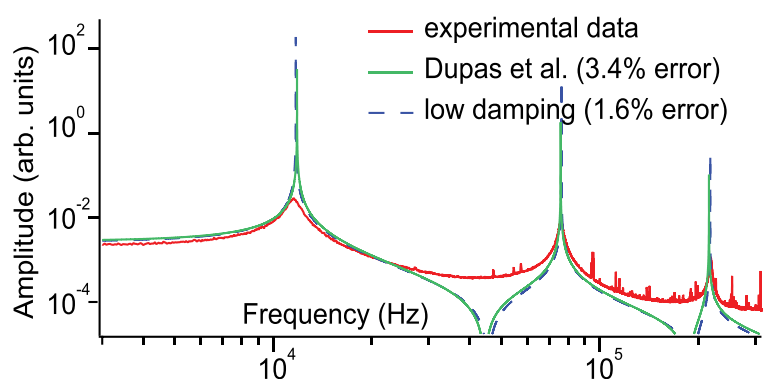

FIG. 2. A typical thermal amplitude spectrum of flexural resonances of the cantilever c1, see Table I, in air. A solid line is a fit of the Dupas model. ${ }^{24} \mathrm{~A}$ dashed line is a more accurate description obtained via Eq. (6). 
resonance frequencies. However, for cantilevers with high quality factors $Q$ of 50, Dupas et al. obtained relative errors of less than $0.5 \%$ for each resonance frequency. This is because the model is essentially fitting the resonances in vacuum and, thus, with no damping. The cantilevers used here have modest quality factors of $10-15$ in air, ${ }^{40}$ so air damping cannot be neglected.

In the limit of $Q \gg 1$, Sader $e \mathrm{al}^{27}$ developed a correction to the resonance frequencies of the AFM cantilevers due to low damping by a hydrodynamic flow

$$
\frac{\omega_{n}^{\text {vac }}}{\omega_{n}^{\text {fluid }}}=\left(1+\frac{\pi \rho_{\text {fluid }} b}{4 \rho t} \Gamma_{r}\right)^{(1 / 2)},
$$

where: $\rho_{\text {fluid }}=\rho_{\text {air }}=1.18 \mathrm{~kg} / \mathrm{m}^{3}$ is air density; $\omega_{n}^{\text {fluid }}$ and $\omega_{n}^{v a c}$ are angular frequencies of the $n-t h$ resonance mode of the AFM cantilever in fluid (here: air) and vacuum, respectively; and $\Gamma_{r}$ is the real part of the hydrodynamic function $\Gamma_{\text {rect }}$ from the footnote (20) in Ref. 27.

The values of $\Gamma_{r}$ apply to non-ideal rectangular cantilevers with an aspect ratio of 3.9 and more. ${ }^{25}$ Thus, they are almost applicable to our cantilevers, which have an aspect ratio of $3.3 \pm 0.1$. Consequently, we upgrade the model of Dupas et al. by using the results of Sader et al. ${ }^{27}$ To do so, we need to translate the corrections in resonance frequencies from Eq. (4) into a wave vector $\kappa$ from Eq. (3). From Eqs. (1)-(3), we find

$$
\kappa=\left(\omega_{n}^{v a c}\right)^{(1 / 2)}\left(\frac{\mu}{E I}\right)^{(1 / 4)} .
$$

Combining Eqs. (4) and (5), we calculate the wave vector $\kappa_{\text {fluid }}$ in the arbitrary fluid

$$
\kappa_{\text {fluid }}=(4 \pi f / t)^{(1 / 2)}\left(\frac{3 \rho}{E}\right)^{(1 / 4)}\left(1+\frac{\pi \rho_{\text {fluid }} b}{4 \rho t} \Gamma_{r}\right)^{(1 / 4)} .
$$

Here, $\omega_{n}^{\text {fluid }}=2 \pi f$ with $f$ being frequency, and $I=t^{3} b / 12$.

The dashed line in Fig. 2 plots the results of the model of Dupas et al. with low hydrodynamic damping, i.e., using $\kappa_{\text {fluid }}$ from Eq. (6) in air. Excellent agreement with the experimental data is obtained and we extend this analysis to three more BL-RC150VB cantilevers itemized as c2 to c4 in Table I. Errors accumulated over their fitted resonance frequencies are $2 \%$ to $6 \% .^{35}$

We now want to fit the resonance frequencies in the PBS buffer. To start with, the dashed line in Fig. 3 plots the results of our upgraded model of Dupas et al. with Eq. (6), where $\kappa_{\text {fluid }}=\kappa_{P B S}$, and density of PBS $\rho_{P B S}=998 \mathrm{~kg} / \mathrm{m}^{3} .{ }^{41,47}$

TABLE I. Properties of the cantilevers used in this study.

\begin{tabular}{lccccccc}
\hline \hline Cantilever & $\begin{array}{c}L \\
(\mu \mathrm{m})\end{array}$ & $\begin{array}{c}b \\
(\mu \mathrm{m})\end{array}$ & $\begin{array}{c}t \\
(\mathrm{~nm})\end{array}$ & $\begin{array}{c}h_{\text {tip }} \\
(\mu \mathrm{m})\end{array}$ & $\begin{array}{c}m_{\text {tip }} \\
(\mathrm{pg})\end{array}$ & $\begin{array}{c}\rho \\
\left(\mathrm{kg} / \mathrm{m}^{3}\right)\end{array}$ & $\beta$ \\
\hline $\mathrm{c} 1$ & 99.6 & 30.2 & 179 & 10.0 & 102 & 7856 & 0.91 \\
$\mathrm{c} 2$ & 100.7 & 31.1 & 174 & 10.0 & 104 & 7993 & 0.97 \\
$\mathrm{c} 3$ & 100.5 & 31.1 & 177 & 9.5 & 101 & 7910 & 0.95 \\
$\mathrm{c4}$ & 100.4 & 30.2 & 174 & 10.0 & 101 & 7993 & 0.91 \\
\hline \hline
\end{tabular}

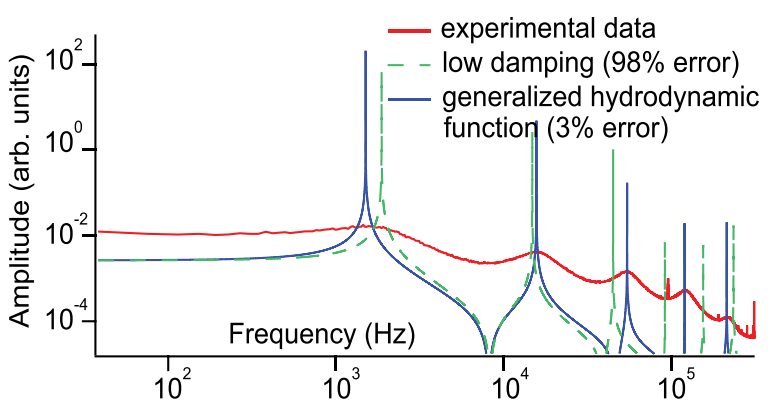

FIG. 3. A typical thermal amplitude spectrum of flexural resonances of the cantilever $\mathrm{c} 1$ in the PBS buffer. A dashed line is a fit of the model via Eq. (6) applied to PBS. A solid line presents a more accurate description obtained via Eq. (7).

Errors of $98 \%$ are obtained, so a more accurate description is needed. ${ }^{35}$

The quality factors of our cantilevers in PBS are about 1.5 for the first resonance at $1.50 \mathrm{kHz}$ and about 2 for higher resonances. Those quality factors are larger than " 1 ," but an actual hydrodynamic function is expected to differ from $\Gamma_{r}$. Thus, we need to find the generalized hydrodynamic function $\mathcal{H}_{r}$ to substitute for $\Gamma_{r}$ in Eq. (6).

Sader et al. ${ }^{25}$ suggested that for a rectangular cantilever with an arbitrary aspect ratio, an imaginary component of the generalized hydrodynamic function $\mathcal{H}_{i m}$ can be approximated by a power law of the Reynolds number $R e$. The value of $R e=\left(2 \pi f \rho_{P B S} b^{2}\right) /\left(4 \eta_{P B S}\right)$, where $\eta_{P B S}$ is the viscosity of PBS. Thus, we suggest a complementary power law to describe the real component of the generalized hydrodynamic function $\mathcal{H}_{r}$. In order to find $\mathcal{H}_{r}$ in the limit of small damping, we manipulate Eq. (4) to yield $\mathcal{H}_{r}(R e)=\left[\left(\frac{\omega_{p r a c}^{v a c}}{\omega_{n}^{B S}}\right)^{2}-1\right]\left(\frac{4 \rho t}{\pi \rho_{P B S} b}\right)$. The values of $\omega_{n}^{v a c}$ are obtained from the model of Dupas et al. using the cantilever properties from Table I. In addition, due to the lack of hydrodynamic damping in vacuum, we include two more resonances calculated using the model of Dupas et al. at $438 \mathrm{kHz}$ and $727 \mathrm{kHz}$, respectively. These resonances become visible in PBS at $120 \mathrm{kHz}$ and $215 \mathrm{kHz}$ in Fig. 3, respectively. ${ }^{35}$

To visualize the power law dependence of $\mathcal{H}_{r}$ with $R e$, Fig. 4 plots the decimal logarithm of $\mathcal{H}_{r}$ vs. the decimal logarithm of $R e$ for the five resonances observed in PBS and for the cantilevers $\mathrm{c} 1$ to $\mathrm{c} 4$. Our data are best fitted with a quadratic relation, i.e., $\log \mathcal{H}_{r}=a_{0}+a_{1} \log R e+a_{2}(\log R e)^{2}$, which yields: $\mathcal{H}_{r}=A_{0}(R e)^{\left[a_{1}+a_{2}(\log R e)\right]}$ with $A_{0}=10^{a_{0}}$. Numerical values of the fit coefficients are presented in

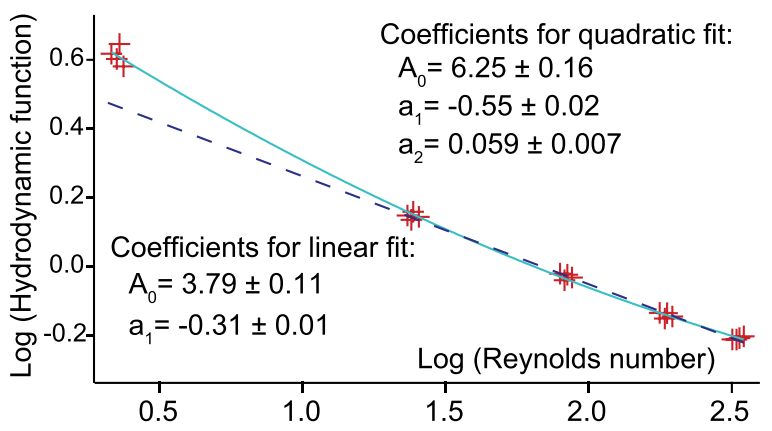

FIG. 4. Log-log plot of $\mathcal{H}_{r}$ vs. $\operatorname{Re}$ for the cantilevers listed in Table I. 
Fig. 4. Fit quality is estimated by calculating the value of $\chi_{\text {red }}^{2}$. ${ }^{42}$ We obtain $\chi_{\text {red }}^{2} \simeq 2 * 10^{-4}$ indicating a very good fit.

We begin our discussion of Fig. 4 by testing statistical significance of the quadratic term, i.e., a statistical hypothesis $H_{0}: a_{2}=0$. We find that with $99 \%$ confidence level $H_{0}$ is not true and $a_{2}$ is not zero. ${ }^{43}$ However, the values of $\log \mathcal{H}$ become linearly dependent on $\log R e$, once we omit the data for the first resonance, i.e., with $\log R e<0.5$. A small value of $\chi_{\text {red }}^{2} \simeq 1 \times 10^{-4}$ suggests a cross-over between two regimes of the hydrodynamic function. Such a cross-over is expected, since the first resonance at $1.50 \mathrm{kHz}$ is the most damped out of all the modes. Thus, hydrodynamic flow is expected to be described by a different functional dependence in the case of the first mode when compared to the other modes. However, with a quadratic fit, we capture a hydrodynamic correction to the wave vector $\kappa_{P B S}$, which applies to both hydrodynamic regimes

$$
\begin{aligned}
\kappa_{P B S}= & \left(4 \pi f_{P B S} / t\right)^{(1 / 2)}\left(\frac{3 \rho}{E}\right)^{(1 / 4)} \\
& \times\left(1+\frac{\pi \rho_{P B S} b}{4 \rho t} A_{0}(R e)^{\left[a_{1}+a_{2}(\log R e)\right]}\right)^{(1 / 4)} .
\end{aligned}
$$

Using the coefficients $A_{0}, a_{1}$, and $a_{2}$ within their errors, we refit the data in Fig. 3 and find only 3\% agglomerated error over five resonances. Extending such analysis to the remaining cantilevers yields errors between $3 \%$ and $11 \% .^{35}$ Propagating the errors from the material and geometrical parameters, we obtain errors between $10 \%$ and $40 \%$ with an average of $20 \%$. $^{35}$

We apply our model to predict the shifts of five resonances in contact with a folded protein molecule in PBS. Supposing that a protein has a normal elastic spring constant $k_{n}$ of about $10 \mathrm{pN} / \mathrm{nm}^{44-46}$ as well as $k_{\text {lat }}=k_{n}$ and $\gamma_{n}=\gamma_{\text {lat }}=10^{-8} \mathrm{~kg} / \mathrm{s}$, see Ref. 45, one obtains well distinguishable $81 \%$ combined shift of the five resonance frequencies. ${ }^{35}$ This is much larger than our average propagated errors of $20 \%$. However, $20 \%$ combined shifts in the five resonance frequencies would affect the values of $k_{n}$ and $\gamma_{n}$ as follows. With $\gamma_{n}=10^{-8} \mathrm{~kg} / \mathrm{s}, k_{n}$ would need to change to either 5 or $15 \mathrm{pN} / \mathrm{nm}$. With $k_{n}=10 \mathrm{pN} / \mathrm{nm}, \gamma_{n}$ would need to change to either $2 \times 10^{-8}$ or $10^{-9} \mathrm{~kg} / \mathrm{s}$. The values of $\gamma_{n}<10^{-9}$ have no effect on error at $k_{n}=10 \mathrm{pN} / \mathrm{nm}$. The variations in $k_{n}$ and $\gamma_{n}$ are substantial. However, the issue of elastic spring constants and dissipation factors for the proteins is still in its infancy, and it is not clear whether those changes are dramatic or not. ${ }^{46}$ The results of our model can be improved by using SEM measurements of the geometrical properties of AFM cantilevers. ${ }^{35}$ In addition, higher electronics bandwidth of the AFM setup will allow including more resonances of the cantilevers and obtaining lower uncertainties of $k_{n}$ and $\gamma_{n}$.

We expect our results to be transferable to other cantilevers with similar aspect ratio and buffers with similar ionic strengths, e.g., tris-buffered saline. Further studies are needed to account for corrections coming from van der Waals and electrostatic forces in the proximity of BOs. However, once an AFM cantilever is in contact with an arbitrary body, the forces acting in the contact zone typically surpass any non-contact interactions. ${ }^{13,14}$
In conclusion, we have developed an accurate model and a method to fit thermal resonances for compliant AFM cantilevers in biological media like PBS. Greater numbers of observed resonances will provide more precise values of mechanical signatures. Other upgrades need to account for the non-contact corrections for the resonance frequencies of AFM cantilevers in proximity to BOs. Mechanical signatures of proteins and cells can now be obtained by fitting the frequency shifts of flexural resonances of AFM cantilevers in contact with BOs. Our model can also be used to describe changes in the mechanical signature with time, e.g., to describe single protein folding trajectories under force.

The authors acknowledge Govind Paneru and Professor Bret Flanders for instrumental help in optical measurements of the AFM cantilevers.

${ }^{1}$ C. Bustamante, Y. Chemla, N. Forde, and D. Izhaky, Annu. Rev. Biochem. 73, 705 (2004).

${ }^{2}$ M. J. Buehler and T. Ackbarow, Comput. Methods Biomech. Biomed. Eng. 11, 595 (2008).

${ }^{3}$ A. Sorkin and M. von Zastrow, Nat. Rev. Mol. Cell Biol. 10, 609 (2009).

${ }^{4}$ J. Arnadottir and M. Chalfie, Annu. Rev. Biophys. 39, 111 (2010).

${ }^{5}$ L. Han, A. J. Grodzinsky, and C. Ortiz, Annu. Rev. Mater. Res. 41, 133-168 (2011).

${ }^{6}$ N. B. Becker and R. Everaers, J. Chem. Phys. 130, 135102-10 (2009).

${ }^{7}$ P. V. Cornish and T. Ha, ACS Chem. Biol. 2, 53-61 (2007).

${ }^{8}$ J. M. Fernandez and H. B. Li, Science 303, 1674 (2004).

${ }^{9}$ C. Cecconi, E. Shank, C. Bustamante, and S. Marqusee, Science 309, 2057 (2005).

${ }^{10}$ W. J. Greenleaf, M. T. Woodside, and S. M. Block, Annu. Rev. Biophys. Biomol. Struct. 36, 171 (2007).

${ }^{11}$ G. Zoldak and M. Rief, Curr. Opin. Struct. Biol. 23, 48 (2013).

${ }^{12}$ T. Ando, T. Uchihashi, and N. Kodera, Annu. Rev. Biophys. 42, 393 (2013).

${ }^{13}$ B. Cappella and G. Dietler, Surf. Sci. Rep. 34, 1 (1999).

${ }^{14}$ H.-J. Butt, B. Cappella, and M. Kappl, Surf. Sci. Rep. 59, 1 (2005).

${ }^{15}$ R. Szoszkiewicz and E. Riedo, in Applied Scanning Probe Methods V, edited by B. Bhushan, H. Fuchs, and S. Kawata (Springer-Verlag, Heidelberg, 2007), pp. 269-286.

${ }^{16}$ J. Adamcik, C. Lara, I. Usov, J. Jeong, F. S. Ruggeri, G. Dietler, H. Lashuel, I. Hamley, and R. Mezzenga, Nanoscale 4, 4426 (2012).

${ }^{17}$ R. Szoszkiewicz, A. Kulik, G. Gremaud, and M. Lekka, Appl. Phys. Lett. 86, 123901 (2005).

${ }^{18}$ R. Garcia and E. Herruzo, Nat. Nanotechnol. 7, 217 (2012).

${ }^{19}$ J. Lozano and R. Garcia, Phys. Rev. Lett. 100, 076102 (2008).

${ }^{20}$ A. Raman, S. Trigueros, A. Cartagena, A. Stevenson, M. Susilo, E. Nauman, and S. Antoranz-Contera, Nat. Nanotechnol. 6, 809 (2011).

${ }^{21}$ O. Sahin, C. Quate, O. Solgaard, and A. Atalar, Nat. Nanotechnol. 2, 507 (2007).

${ }^{22}$ D. Kiracofe and A. Raman, Phys. Rev. B 86, 205405 (2012).

${ }^{23}$ R. Szoszkiewicz, Rev. Sci. Instrum. 83, 037101 (2012).

${ }^{24}$ E. Dupas, G. Gremaud, A. Kulig, and J.-L. Loubet, Rev. Sci. Instrum. 72, 3891 (2001).

${ }^{25}$ J. Sader, J. Sanelli, B. Adamson, J. Monty, X. Wei, S. Crawford, J. Friend, I. Marusic, P. Mulvaney, and E. Bieske, Rev. Sci. Instrum. 83, 103705 (2012).

${ }^{26}$ J. Chon, P. Mulvaney, and J. Sader, J. Appl. Phys. 87, 3978 (2000).

${ }^{27}$ J. Sader, J. Appl. Phys. 84, 64 (1998).

${ }^{28}$ A. Dey and R. Szoszkiewicz, Nanotechnology 23, 175101 (2012).

${ }^{29}$ The errors are calculated using a formula: $\sum_{i}\left|f_{\text {measured }}^{(i)}-f_{\text {fitted }}^{(i)}\right| / f_{\text {measured }}^{(i)}$, where $f_{\text {measured }}^{(i)}$ and $f_{\text {fitted }}^{(i)}$ are the $i$-th measured and fitted frequencies, respectively.

${ }^{30}$ M. Carrion-Vazquez, A. Oberhauser, T. Fisher, P. Marszalek, H. Li, and J. Fernandez, Prog. Biophys. Mol. Biol. 74, 63 (2000).

${ }^{31}$ H. Dietz, F. Berkemeier, M. Bertz, and M. Rief, Proc. Natl. Acad. Sci. U.S.A. 103, 12724 (2006).

${ }^{32} \mathrm{~A}$ torsional spring constant $k_{\text {tor }}$ with its proper dissipation factor $\gamma_{t o r}$ can be used in addition or instead of $k_{\text {lat }}$ and $\gamma_{\text {lat }}$.

${ }^{33}$ A. Khan, J. Philip, and P. Hess, J. Appl. Phys. 95, 1667 (2004). 
${ }^{34}$ We calculate the Young's modulus using a formula for a two-layer composite beam-Ref. 27 in the paper of Gavan et al. ${ }^{36}$ - comprised of $50 \mathrm{~nm}$ gold and silicon nitride. Thickness of the silicon nitride itself is estimated within $114 \mathrm{~nm}$ to $186 \mathrm{~nm} .{ }^{35}$ Error in the Young modulus is obtained using the results of of Gavan et al., ${ }^{36}$ who measured Young's moduli of thin silicon nitride films.

${ }^{35}$ See supplementary material at http://dx.doi.org/10.1063/1.4858411 for optical and scanning electron microscopy images of AFM cantilevers, application of our model to proteins, and error propagation analysis.

${ }^{36}$ K. Gavan, H. Westra, E. van der Drift, W. Venstra, and H. van der Zant, Appl. Phys. Lett. 94, 233108 (2009).

${ }^{37}$ Frequency of the $1 \mathrm{st}$ torsional resonance is calculated from $f_{\text {tor }}=0.5 \frac{t}{L b}\left(\frac{E}{\rho(2+2 \nu)}\right)^{1 / 2}$, where the Poisson ratio $\nu=0.2$ for a SiNx cantilever is obtained from Ref. 33.

${ }^{38}$ Half a pyramid with a square base was used, with a side $a=b / 4$ to yield the value of $m_{t i p}=\rho(t / 6)\left[(b+4 t)\left(h_{t i p}+2 t\right)+b^{2} / 8\right]$.

${ }^{39}$ Displacements of the support spring of up to several millimeters were correlated with dynamometer's measurements of forces.

${ }^{40}$ The quality factors are estimated from a ratio of the amplitudes on the resonance and at the arbitrarily chosen low frequency.
${ }^{41}$ The PBS density of $998 \mathrm{~kg} / \mathrm{m}^{3}$ was measured in Ref. 47 at temperature of about $22{ }^{\circ} \mathrm{C}$.

${ }^{42}$ J. Taylor, An Introduction to Error Analysis: The Study of Uncertainties in Physical Measurements, 2nd ed. (University Science Books, 1996).

${ }^{43} \mathrm{~A}$ t-Student test is used to test $H_{0}: a_{2}=0$. To do so, a calculated t-Student coefficient for $a_{2}$ is compared with its tabulated value for a given number of degrees of freedom and at a 99\% confidence level. From the data in Fig. 4 , we get a value of $a_{2}=0.059$ and its standard deviation $s_{a_{2}}=0.011$. Thus, the calculated t-Student coefficient is $t\left(a_{2}\right)=a_{2} / s_{a_{2}}=5.4$. This value is larger than a tabulated value $t(17 ; 0.01)=2.9$ read from the statistical tables for $17^{\circ}$ of freedom and at $99 \%$ confidence level (P. Bevington, Data Reduction and Error Analysis for the Physical Sciences (McGraw-Hill Book Company, New York, 1969). Thus, $\mathrm{H}_{0}$ is not accepted.

${ }^{44}$ Y. Wang and G. Zocchi, EPL 96, 18003 (2011).

${ }^{45}$ Y. Taniguchi, B. S. Khatri, D. J. Brockwell, E. Paci, and M. Kawakami, Biophys. J. 99, 257 (2010).

${ }^{46}$ K. E. Malek and R. Szoszkiewicz, "Changes of protein stiffness during folding detect protein folding intermediates," J. Biol. Phys. (in press). DOI: $10.1007 / \mathrm{s} 10867-013-9331-y$.

${ }^{47}$ J. Schiel and D. Hage, Talanta 65, 495 (2005). 